\title{
The Effect of Route-choice Strategy on Transit Travel Time Estimates
}

\author{
Nate Wessel ${ }^{1}$ and Steven Farber ${ }^{2}$ \\ 1 Department of Geography and Planning, University of Toronto \\ 2 Department of Human Geography, University of Toronto Scarborough
}

April 26, 2019

\begin{abstract}
Estimates of travel time by public transit often rely on the calculation of a shortest-path between two points for a given departure time. Such shortest-paths are time-dependent and not always stable from one moment to the next. Given that actual transit passengers necessarily have imperfect information about the system, their route selection strategies are heuristic and cannot be expected to achieve optimal travel times for all possible departures. Thus an algorithm that returns optimal travel times at all moments will tend to underestimate real travel times all else being equal. While several researchers have noted this issue none have yet measured the extent of the problem. This study observes and measures this effect by contrasting two alternative heuristic routing strategies to a standard shortest-path calculation. The Toronto Transit Commission is used as a case study and we model actual transit operations for the agency over the course of a normal week with archived AVL data transformed into a retrospective GTFS dataset. Travel times are estimated using two alternative route-choice assumptions: 1) habitual selection of the itinerary with the best average travel time and 2) dynamic choice of the next-departing route in a predefined choice set. It is shown that most trips present passengers with a complex choice among competing itineraries and that the choice of itinerary at any given moment of departure may entail substantial travel time risk relative to the optimal outcome. In the context of accessibility modelling, where travel times are typically considered as a distribution, the optimal path method is observed in aggregate to underestimate travel time by about 3-4 minutes at the median and 6-7 minutes at the $90^{\text {th }}$ percentile for a typical trip.
\end{abstract}




\section{Introduction}

Accessibility metrics are widely used in transport planning, where accessibility is defined as a measure of the relative ease of travelling to some set of destinations distributed around a region. Accessibility is concerned not only with where people do go, but also with where they might go and is often thought of as a measure of people's freedom or ability to engage with opportunities, procure services, and generally engage with the life of a city or region. Accessibility is typically estimated separately for different modes of transport and this study is concerned with accessibility by public transport, hereafter referred to as transit. Travel by transit has relatively low monetary costs, and transit accessibility studies have tended to emphasize travel time as the main or only component of accessibility measures (e.g. Owen and Murphy, 2018a). The estimation of accurate transit travel times is thus critical to an accurate measure of transit accessibility.

While accurate estimates of travel time can in general be derived from various techniques for observing a sample of actual travellers, such techniques are not able to be generalized well to the problem of estimating potential travel times. Rather than observe real passengers transit accessibility researchers have relied instead on modelling transit systems using schedule data which has become widely available (See e.g. Zervaas, 2018) in a standard format known as the General Transit Feed Specification (GTFS). Such models, among other problems (See Wessel and Farber, 2019), do not realistically simulate passenger route choice. While some transit trips may have only one obvious way of getting from A to B, many others present passengers with several competing alternatives. Given the stochastic nature of transit operations, it is impossible to know a priori which will minimize travel time, and a real passenger is required to somehow make a decision with limited information which will impact their realized travel time. Rather than model this decision process, current GTFS-based models have relied on algorithms which return the minimum possible travel time for any given moment of departure - even where the choice of an optimal itinerary would be extremely complex and require information which is not available to an actual passenger.

This paper argues that more realistic assumptions about route-choice are needed in transit accessibility models and makes the case for this by demonstrating that route selection heuristics have a substantial impact on estimated travel times. We take the Toronto Transit Commission (TTC) as a case study and model transit operations with a retrospective GTFS dataset (Wessel, Allen, and Farber, 2017) describing the actual operations of the agency over the course of five

weekdays. Using this dataset, we calculate travel times using both a standard shortest-path method and using two alternative heuristic strategies. One strategy is based on an assumption that people are creatures of habit, the other on an assumption that they may vary their choice based on real-time information at the time of departure. By observing the absolute differences in travel times between the shortest path and heuristic estimates we can then quantify the degree to which 
current models of accessibility may underestimate travel times and thus over-estimate accessibility. We also seek to provide context for the eventual development of better route-selection heuristics by quantifying the choice scenario faced by passengers. To this end we propose an entropy-based measure of route-choice complexity and quantify the travel time risk associated with route choice at any given moment of departure.

Section 2 provides context for this study and further develops the conceptual problem of routechoice in stochastic transit networks. Section 3 lays out the methods and data used, providing a detailed illustration of the two tentative route-selection heuristics. Finally, Section 4 presents the results and Section 5 summarizes the findings.

\section{Literature Review}

\subsection{Transit Accessibility}

In transport research, accessibility is a relative measure of the ease with which some set of spatially distributed destinations can be reached by a potential traveller. In studies of transit specifically,

this "ease" has typically been defined as a function of travel time only, though other cost functions have been developed. For example El-Geneidy et al. (2016) included the monetary fare price to account for the experiences of low income transit users - and Cui and Levinson (2018) propose a general (not specific to transit) measure of accessibility that incorporates external costs of travel such as insurance, pollution, and public subsidy of infrastructure. Nassir et al. (2016) propose a utility-based accessibility function, attempting to measure the subjective experience of using transit. They even consider route choice in this context, though only in as much as multiple route options are theorized to present travellers with more options on which to exercise their preferences.

Despite such efforts, the literature on transit accessibility is dominated by the use of estimated travel time as the only measure of accessibility (e.g. Farber and Fu, 2017; Boisjoly and El-Geneidy, 2016; Ma and Jan-Knaap, 2014; Owen and Levinson, 2016; Owen and Murphy, 2018b; J. Lee and Miller, 2018; Wessel and Farber, 2019; Conway, Byrd, and Linden, 2017). As the internal monetary costs of using transit are relatively low, and the conversion between time and money inexact at best, this is not likely to change in the near future, nor do we suggest that it should. Travel time is likely to remain the largest impediment to travel that transit users encounter.

\subsection{Estimating Travel Times}

Given the dominance of travel time in the measure of accessibility, the accurate estimate of such times is critical to the study of transit accessibility. 
Probably the most accurate way to estimate travel times on transit is to actually observe passengers making trips and see how long they take to reach a destination. In transit systems with smart-cards and distance-based fares, this can be possible if passengers are required to tap their card both when entering and leaving the system (Sun and Xu, 2012). Systems such as these can produce huge volumes of trip time measurements (Jang, 2010) and travel times between stations or stops can often be inferred quite easily by taking the difference between tap-in and tap-off times (H. Lee et al., 2017). When observed over time these systems can be expected to generate distributions of travel times that accurately characterize actual travel between stations. While fare systems like these can be extremely useful for service planning, they are relatively uncommon outside of Asia, except where they are applied only to rapid transit (e.g. WMATA) or commuter rail systems (e.g. GO Transit), but not the connecting bus/streetcar networks. In any case, such data are limited in their potential applicability to accessibility metrics by their reliance on observed travel behaviour. Pairs of stops that are not well frequented may have few or no observations connecting them.

Similarly, several methods have been employed for observing transit passengers using connected mobile devices, either by recording their movements with GPS (Stenneth et al., 2011) or detecting their presence with Bluetooth sensors (Dunlap et al., 2016). Such datasets would almost certainly produce a smaller sample of observed trips than the smart-card data, and would suffer from the same issue - that trips not commonly made cannot be observed.

By far the most common method of estimating travel times for the purpose of measuring accessibility however is to use an application which models the transit system from standardized schedule-based GTFS data, which has become very widely available in recent years (Zervaas, 2018). Some such applications were designed with passenger navigation assistance in mind, as with OpenTripPlanner (OpenTripPlanner Project, 2019), while others such as R5 (Conway, Byrd, and Linden, 2017) and UrbanAccess (Blanchard and Waddell, 2017) were developed explicitly for the purpose of transit accessibility analysis.

These applications take two different approaches to travel time estimation. OpenTripPlanner and R5, along with ArcMap's GTFS Network Analyst (Morang, 2019) closely follow the data model defined by the GTFS and integrate it with a pedestrian network e.g. from OpenStreetMap. Each transit vehicle scheduled to pass from one stop to the next is modelled as a directed edge in a large space-time graph comprising transit trips, stops, and walking edges. This graph is then scanned by shortest-path algorithms such as RAPTOR (Delling, Pajor, and Werneck, 2014) or Dijkstra's Algorithm. By contrast, UrbanAccess takes a faster, more heuristic approach, pre-processing the GTFS data into an essentially atemporal network graph, where travel times and waiting times are summarized as averages and associated with transit edges (Blanchard and Waddell, 2017). This approach, while extremely fast and useful in many circumstances, has substantial limitations such 
as the inability to consider timed arrivals, headway and speed variability, and variability over time (Conway, Byrd, and Linden, 2017). The more detailed approach has its own drawbacks however. For example it produces false precision by assuming that schedule data is accurate (Wessel and Farber, 2019) and as Conway, Byrd, and Linden (2017) note regarding R5:

The accessibility calculations assume that people have perfect information and choose the optimal path to reach their destination given their departure time.

This latter issue has been noted elsewhere in the accessibility literature as well (Morang, 2019; Wessel and Farber, 2019; Kaplan et al., 2014) but to date has not been addressed directly.

\subsection{Retrospective GTFS}

Some of the most recent work in transit accessibility has addressed the assumption that GTFS schedule data is an accurate representation of transit operations. Wessel, Allen, and Farber (2017) outline a method for constructing a retrospective GTFS dataset based on an historic archive of automatic vehicle location (AVL) data. This retrospective GTFS archive then contains not expected future but rather observed past arrival times, and by virtue of its identical format can be used in the same types of network level analysis to which schedule-based GTFS is applied. A similar technique was used by Stewart (2017) to produce more realistic travel-time accessibility metrics applied in the context of London, England and Boston, Massachusetts. Wessel and Farber (2019) generalize the problem and assess the accuracy of schedule-based accessibility in the general case by studying four different North American transit agencies over the course of a week and comparing the differences between retrospective and schedule-based accessibility outcomes. They find that schedule-data over-estimate access by about 5-15\% across the board and that there is a great deal of spatial variation with some zones differing very substantially from the expected access score.

However in their use of retrospective GTFS these applications make the same problematic assumption of "perfect information" raised in the previous section all the more pressing. When shortest path routing is done on a retrospective GTFS package, one not only assumes that passengers have perfect knowledge of a schedule but that they have perfect knowledge of the actual future locations of all transit vehicles, including effects of traffic congestion, breakdowns, short-turns, etc. The shortcomings of this assumption have not yet been directly addressed.

\subsection{Route Choice}

Studies of route choice in transit have made clear that actual passengers do not behave in quite the same way as algorithms when selecting routes. While passengers have been observed in general 
to prefer routes that minimize travel time, walk distance, and number of transfers (Jánošíková, Slavík, and Koháni, 2014; Eluru, Chakour, and El-Geneidy, 2012), it has not been demonstrated that they are able to optimize these preferences very effectively from moment to moment. Rather than suppose that passengers have all the necessary information and are rational decision makers (See Gärling, 1998), we emphasize the role of habit formation and strategy development.

The role of habit in travel behaviour has been much discussed (See Gärling and Axhausen, 2003) and it seems reasonable to suppose that passengers making regular trips learn from their experiences and form habits which they perceive as generally optimal regarding mode choice, route choice, etc. While habit in the context of route choice specifically has not been well studied, some evidence does point to it's influence. Kim, Corcoran, and Papamanolis (2017) observe passengers in Brisbane, Australia and measure the apparent extent of habit in their route choice behaviour. They find that passengers seem more likely to stick to one of several possible routes when they are more frequent travellers (e.g. commuters), travelling between familiar O/D pairs, and when there are substantial potential time differences between the alternatives.

Schmöcker et al. (2009) emphasize the potential role of strategy in route choice, suggesting that passengers engage in consistent strategies such as taking the first arriving vehicle from a set of available options. While the routing strategy may be consistent, the actual route outcomes observed can vary widely. They term the set of paths potentially derived from such a strategy the hyperpath. Kurauchi, Schmöcker, Shimamoto, et al. (2014) find evidence of hyperpath-style routing among regular bus commuters in London, England. They speculate that such variability may be due to strategies such as taking the first bus to arrive (See Kurauchi, Schmöcker, Fonzone, et al., 2012) or selecting the fastest from multiple overlapping lines which would reach the destination.

\section{Methods and Data}

Our purpose in this research is to measure the size of the effect that the assumption of perfect information may have on travel time estimates. We do this by comparing standard "perfect information" travel time estimates to those based on two alternative assumptions:

1. Passengers select a route which is generally the best and stick with it as a matter of habit

2. Passengers use a strategy based on available real-time information, taking the next departing vehicle toward their destination from a set of known alternatives

In either case the resulting travel time estimates must be equal or higher and thus accessibility equal or lower than if the passenger were supposed to act with perfect information. We wish to find out how large this potential gap is and explore any consistent patterns in it's distribution. We hypothesize that there is a substantial effect on travel time and that trips where the passenger 
would face a larger set of route choices will tend to be most overestimated in the perfect information scenario. We further expect that more route choices will be available on longer trips and trips that run diagonal to the street grid, thus requiring transfers.

\subsection{Case Study - Data}

We take as our case study the Toronto Transit Commission (TTC), which operates a large, grid-like network of high frequency transit lines spanning the City of Toronto, Ontario. It is the experience of the authors that this network often produces difficult and unsatisfying route choices, as many potential trips are served by multiple lines, few of which adhere closely to a published schedule, and many of which exhibit substantial variation in headway and in-vehicle travel time.

To accurately represent this variability we make use of a retrospective GTFS package describing the state of the transit network for the five weekdays of November $6^{\text {th }}-10^{\text {th }}, 2017$. A retrospective GTFS, as outlined by Wessel, Allen, and Farber (2017) is a dataset in GTFS format that describes the exact operations of a transit agency as they were, based on observed transit vehicle locations, rather than as they were scheduled to be. The time period in question was a normal week in Toronto, marked neither by major holidays nor weather events and we assume it is representative of typical levels of service for the TTC. The dataset, and the code used to produce it are made available online.

A limitation of this dataset is that AVL data were not available for the subway system - the four subway lines (See Figure 1) are modelled in the retrospective GTFS according to the schedule. This will have the effect of artificially reducing service variability on these lines, though in reality these are likely already the least variable, as they are the only lines the TTC operates in a fully grade-separated right of way.

\subsection{Measurement Strategy}

To calculate an accessibility measure based on travel times, one must define first, a set of trips from origins to destinations (ODs) and second, an impedance function specifying the utility of a destination given the travel time between ODs.

Defining a set of ODs is necessarily application specific, though some researchers have adopted the convention of measuring residential access to jobs when discussing accessibility measures in abstracto (e.g. Allen and Farber, 2018; Owen and Levinson, 2016). We take a different approach here, adopting a spatially uniform sampling strategy and giving roughly equal weight to all places served by transit in the city. All parts of Toronto are quite dense as the city is at the center of a larger region and this strategy has the benefit of remaining agnostic to the distribution of activities one may wish to study. It also does not place undue emphasis on access to the extreme 
density of jobs in the downtown core $^{1}$.

Similarly, the choice of an impedance function may be guided by application, or for methodological studies by convention. At present however the literature on accessibility is quite active and no consensus has been established on impedance metrics that are appropriate in this context. Some measures like "cumulative opportunities" may be commonly used but are poorly justified by theory or observation. Many other methods are better justified by theory but not yet tested empirically or widely applied. To make the results of this study relevant to ongoing innovation in this area, we will not calculate accessibility as such but rather look directly at the travel times used to construct any such metric. Our hope is that researchers will be able to infer the implications of absolute changes in travel times for their own preferred impedance function. As all reasonable impedance functions share an interest in modelling, with more or less accuracy, reasonable human travel behaviour we can make our observations most relevant to the field by selecting for observation trips which have lengths characteristic of actual travel.

The goal then is to generate a set of trips which is spatially random yet representative of typical trip lengths for the region. This is accomplished by defining a large set of dispersed OD points, weighting the population of potential trips between them to be representative of typical travel behaviour in the city, and taking a weighted random sample from the population.

We define the set of possible ODs as the intersections of all major transit lines in the city. 440 points were manually placed at these locations with some exceptions where points would otherwise be very close together (Figure 1). Given that the density of the resulting point pattern exhibits some spatial variation, we further approximate a spatially uniform sample by assigning weights to each point based on a rough approximation of its catchment area. To measure this we constructed a Voronoi tessellation of the points and clipped it to a $1 \mathrm{~km}$ buffer. The area of each catchment is applied as a weight to the points and weights are assigned to OD pairs as the product of the origin and destination weights.

The City of Toronto is quite large and a simple spatially random sample was skewed toward atypically long trips, as shown in Figure 2. To correct for this, we iteratively re-weighted all potential OD pairs in the sample according to the distance between them such that the probability density function (PDF) of weighted trip lengths matched a PDF of actual trips made on the TTC within the City of Toronto. Distance was estimated with Manhattan distance between ODs accounting for the $\approx 17$ degree rotation of most of the street network from true north. The empirical distribution of trip lengths was derived from the 2016 Toronto Transportation Tomorrow Survey (TTS, 2019). A weighted random sample of 1,000 OD pairs was taken from the population

\footnotetext{
${ }^{1}$ The quality of the retrospective GTFS data is poorest downtown due to 1) relatively poor GPS signal around tall buildings in the downtown core and 2) the predominance of the subway in this part of the city, which as we've noted is based only on schedule data.
} 
of all possible OD combinations. Figure 2 provides a comparison of the distribution of trip lengths between the sample and the empirical dataset.

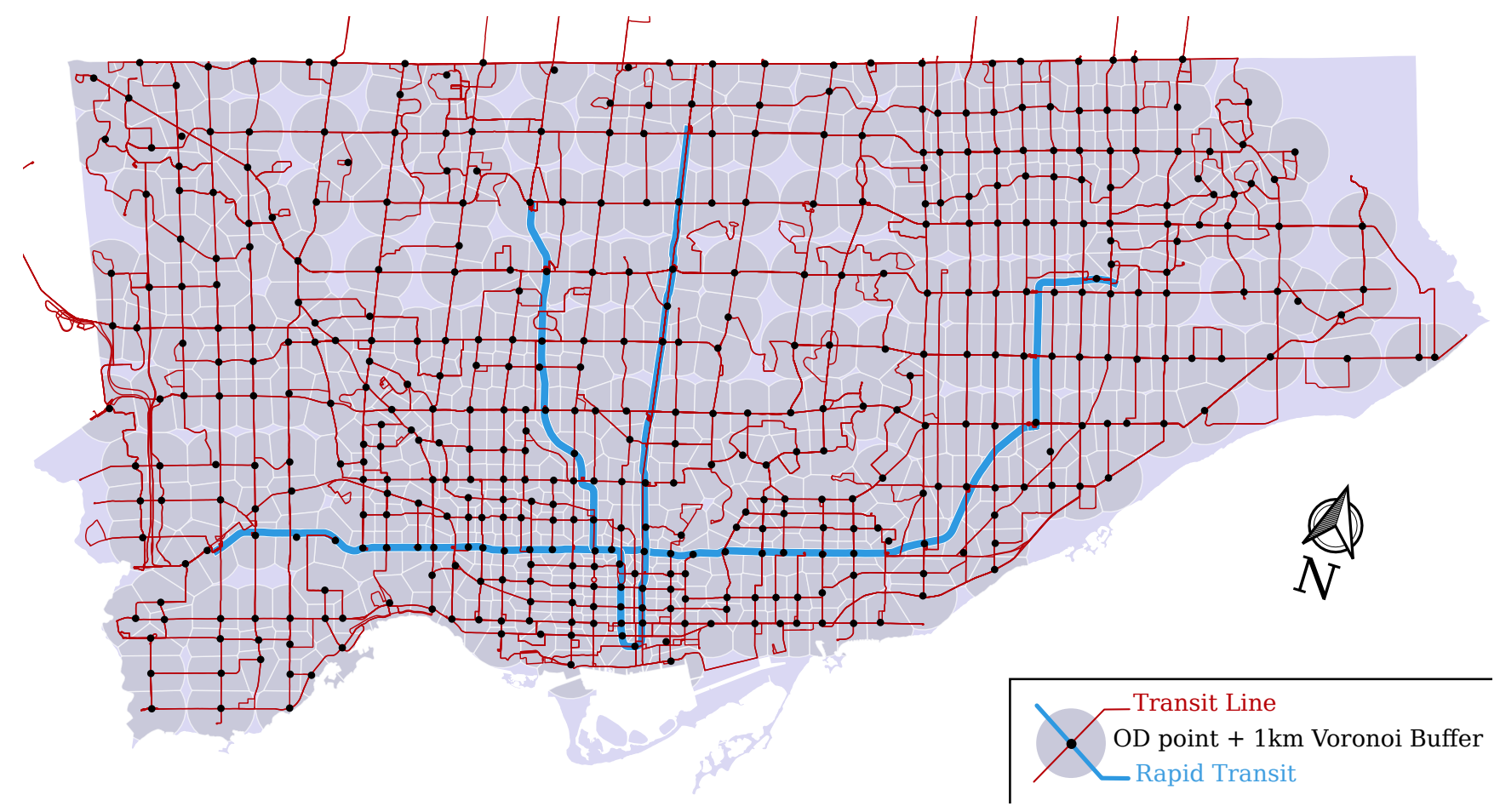

Figure 1: 440 OD points were placed at major transit intersections and transfer points.

\section{Sample vs. Empirical Trip Lengths}

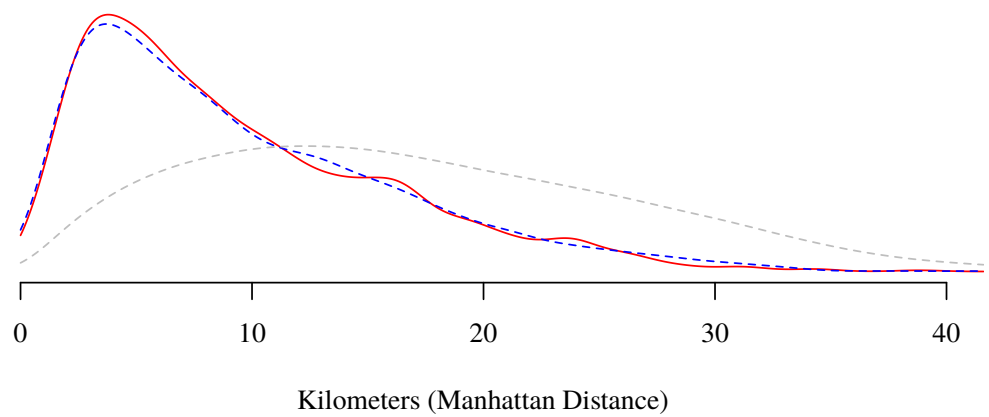

Figure 2: Distribution of trip lengths in the sample (red) versus the distribution of actual trips based on the Toronto Travel Survey (blue). The unweighted distribution of all possible OD pairs joining the sample points is given in grey.

\subsection{Itinerary Choice Set Generation}

We define an itinerary as a set of sequential walking and transit legs connecting an OD pair. Figure 3 provides a simple illustration with three possible itineraries, $a, b$ and $c$. Itinerary $a$ 
involves walking up to stop 1, taking routes 1 or 2 to stop 2 and walking to the destination. Itinerary $b$ instead goes down to route 3 , taking it between stops 3 and 4 , while itinerary $c$ has the user walk the entire route instead of waiting for transit. Walking segments are based on shortest-path network distance.

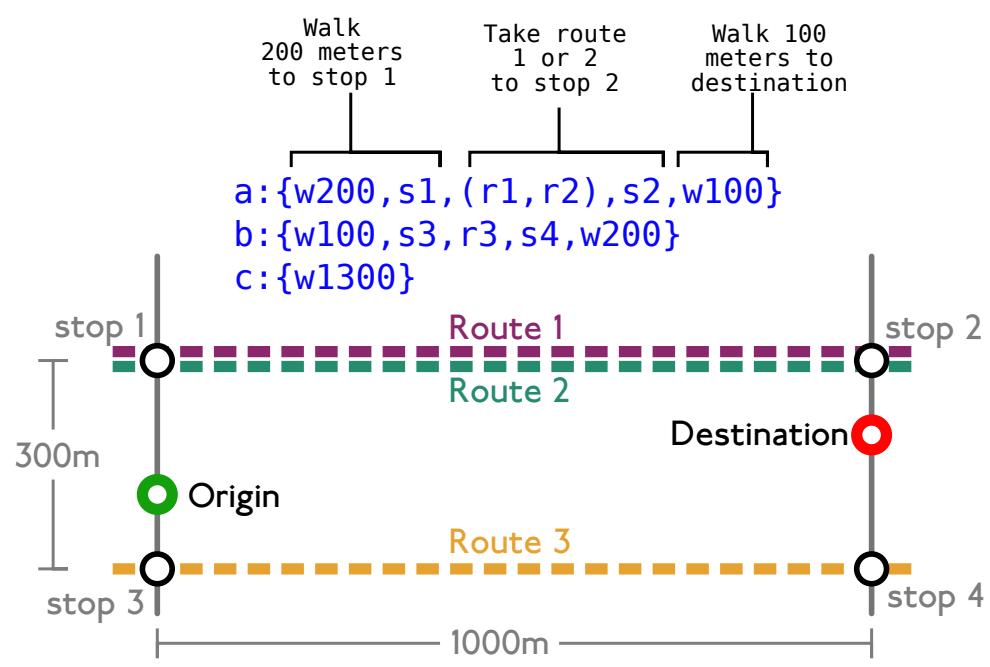

Figure 3: Three possible itineraries $(a, b, c)$ on a simple OD pair. Routes serving the same stop pairs are treated indifferently.

To generate a set of itineraries we use OpenTripPlanner to calculate shortest paths between ODs on the retrospective GTFS data at every minute of the day. For each departure, OpenTripPlanner returns a sequence of walking and transit legs, where transit legs have a start and end stop_id as well as a route_id. Many of the sequences thus generated exhibit minor variations in stop location or route selection. We cluster these various observations into distinct itineraries by treating as equivalent any path which has the same sequence of routes, but different stops or the same sequence of stops but different routes. The first condition can occur due to minor variations in the timing of arrivals at adjacent stops and the latter occurs often where two or more lines share a portion of their route. Itineraries are then defined by the specific details of the most frequent constituent observation. This clustering is done to prevent arbitrary distinctions between essentially identical options.

We break the day into four periods corresponding to general changes in weekday service levels for the TTC (Table 1). For each service period and OD pair, we define the set of potential itineraries as those which are the fastest alternative at any point in the service period across the five days for which we have data. 
Table 1: Distinct weekday TTC service periods (Toronto Transit Commission, 2019).

\begin{tabular}{r|l} 
Service Periods & Time \\
\hline Morning peak & $6 \mathrm{am}-9 \mathrm{am}$ \\
Midday & $9 \mathrm{am}-3 \mathrm{pm}$ \\
Afternoon peak & $3 \mathrm{pm}-7 \mathrm{pm}$ \\
Early Evening & $7 \mathrm{pm}-10 \mathrm{pm}$
\end{tabular}

\subsection{Measuring Route-choice Complexity}

OD pairs which yield larger, more complex choice-sets are expected to produce situations in which it is more difficult for passengers to achieve an optimal travel time. To measure this, we borrow the concept of entropy from the field of information theory. For background on the concept of entropy in information theory see Gray (2011). By treating the optimal itinerary at any given moment as the discrete outcome of a stochastic process, we can see that the problem is similar to that of measuring the information content of a stream of characters. We define entropy, $S$, in Equation 1 where $P_{i}$ is the probability of an itinerary in the choice set being optimal at any given moment in a service period.

$$
S=-\sum_{i} P_{i} \log _{2} P_{i}
$$

The use of a base-2 logarithm means that entropy is measured in bits. A value of 0 bits corresponds to 1 possible itinerary only, 2 bits to two equally likely itineraries, and so on.

\subsection{Route Selection Strategies}

Individuals engage in a wide variety of strategies for selecting transit routes. It is not our purpose here to model this variety accurately - only to suggest some tentative alternatives to the problematic assumption that passengers can optimize their travel time based on perfect information of the system. We define two alternatives based on an assumption of imperfect information.

The first, habitual route choice, we define as the selection of a single itinerary which minimizes mean travel time over a given time window. While our dataset covers only a week, we make the assumption that the service was typical and that a passenger could have learned by experience from past weeks that were essentially identical in terms of travel time performance. As travel time distributions tend to be skewed toward long trips (Xue et al., 2011) the strategy of minimizing a mean also tends to give preference to more reliable itineraries.

For the real-time strategy we assume a passenger has perfect information only about the arrival time of vehicles in the first leg of their itinerary and seeks to minimize their initial wait and walking time where waiting and walking are treated equivalently. This could be approximately the case for 
example if a passenger checked accurate real-time departure estimates before starting their trip. A passenger is assumed to take the itinerary which would let them board a vehicle toward their destination as soon as possible, from the set of all possible itineraries. Where itineraries share a common first leg, and thus a common departure time, the one with the better mean travel time is pursued to the destination.

Finally, we calculate travel times for the perfect information scenario. While these were initially generated with OpenTripPlanner as part of the process of identifying itineraries, we chose to recalculate these for methodological consistency with the two alternatives ${ }^{2}$. We define the perfect information scenario as using only itineraries in the choice set defined for the other alternatives. Unlike the habitual alternative, route choice can vary from moment to moment and unlike the real-time strategy, no itinerary is ever used which would result in a sub-optimal arrival time.

\subsection{An Applied Example}

In this section we illustrate our method by applying it to the first author's morning commute of about 4.6 kilometers. OpenTripPlanner generated five distinct itineraries for this OD pair during the 6-9am morning peak period over the five days for which we have data. Table 2 provides details of each itinerary along with mean travel times and values of $P_{i}$. These correspond to Figure 4, which shows minute-by-minute variation in travel times for each itinerary along with the optimal itinerary at any given moment of departure.

Table 2: Stats for each itinerary illustrated in Figure 4. Entropy for this OD pair is $S=1.67$ for the AM-peak period. Figure 3 indicates how itineraries are to be interpreted.

\begin{tabular}{rlrc} 
Label & Itinerary & $\overline{\text { time }}$ & $P_{i}$ \\
\hline$a$ & $\{\mathrm{w} 137, \mathrm{~s} 3177, \mathrm{r} 47, \mathrm{~s} 3184, \mathrm{w} 57, \mathrm{~s} 14477, \mathrm{r} 2, \mathrm{~s} 14482, \mathrm{w} 828\}$ & 31.5 & 0.494 \\
$b$ & $\{\mathrm{w} 137, \mathrm{~s} 3177, \mathrm{r} 47, \mathrm{~s} 3184, \mathrm{w} 57, \mathrm{~s} 14477, \mathrm{r} 2, \mathrm{~s} 14482, \mathrm{w} 2, \mathrm{~s} 9557, \mathrm{r} 510, \mathrm{~s} 9560, \mathrm{w} 344\}$ & 33.3 & 0.338 \\
$c$ & $\{\mathrm{w} 137, \mathrm{~s} 3177, \mathrm{r} 47, \mathrm{~s} 3180, \mathrm{w} 54, \mathrm{~s} 9600, \mathrm{r} 506, \mathrm{~s} 9614, \mathrm{w} 583\}$ & 36.3 & 0.047 \\
$d$ & $\{\mathrm{w} 137, \mathrm{~s} 3177, \mathrm{r} 47, \mathrm{~s} 3184, \mathrm{w} 57, \mathrm{~s} 14477, \mathrm{r} 2, \mathrm{~s} 14479, \mathrm{w} 28, \mathrm{~s} 5525, \mathrm{r} 94, \mathrm{~s} 5533, \mathrm{w} 217\}$ & 37.7 & 0.107 \\
$e$ & $\{\mathrm{w} 838, \mathrm{~s} 9600, \mathrm{r} 506, \mathrm{~s} 9614, \mathrm{w} 583\}$ & 38.8 & 0.014
\end{tabular}

Itineraries $a, b, c, \& d$ share a common first leg, namely walking to a nearby stop and catching the \#47 bus northbound. These four options diverge from there, with $c$ transferring to the next line, the \#506 streetcar, after only two stops, and $a, b, \& d$ proceeding further up to the subway (Line \#2). $a, b, \& d$ then differ on whether they take the subway all the way to the closest station

\footnotetext{
2 Travel times were estimated from the retrospective GTFS data using software which has been made available online at https://github.com/Nate-Wessel/routing. We did not estimate walking times independently but relied on walking distances between transit legs returned by OpenTripPlanner.
} 


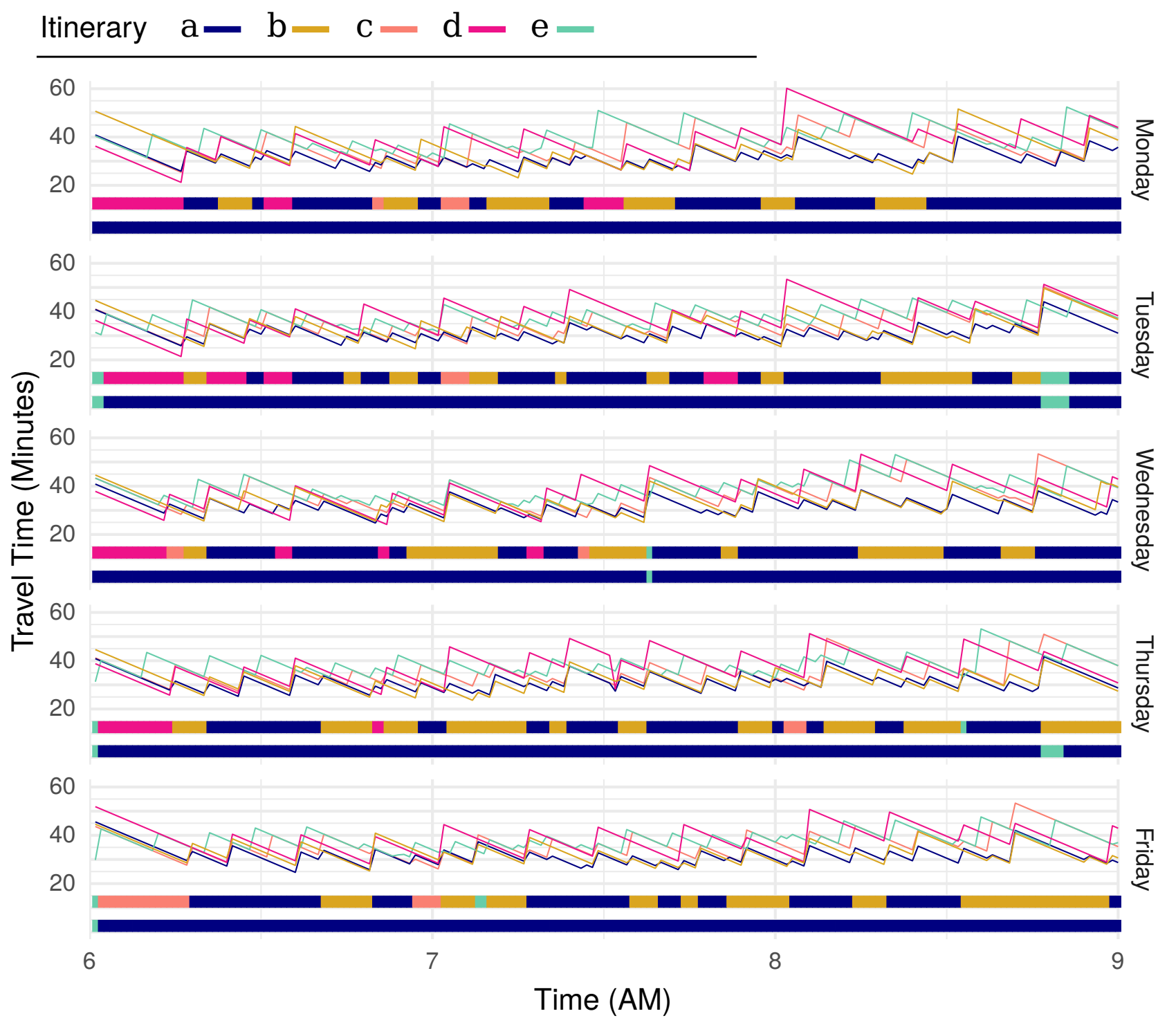

Figure 4: Travel time (including initial wait) for an OD pair varies across five possible itineraries between 6-9AM. The two colored bars across the bottom of each plot indicate the optimal itinerary (top) and the real-time strategy (bottom). The habitual strategy is constant across time and is not shown.

( $a$ ) or reduce the necessary walk by transferring to another line $(b \& d)$ from either of two stations. It's interesting to note that itinerary $d$, which involves a relatively long diversion from the subway to the \#94 bus is only optimal in the earliest part of the period, reflecting increasing congestion on the street that line operates on. Itinerary $b$ is not effected by this, as the \#510 streetcar operates in a partially separated right of way. Itinerary $e$ by contrast to the previous alternatives disregards the \#47 bus, instead walking a few hundred meters of the route up to the \#506 streetcar and taking it the rest of the way.

Itinerary $a$ produces the lowest mean travel time of the alternatives at 31.5 minutes, making 
it the consistent choice of the habitual strategy. As four of the five itineraries share the same first leg, the real-time strategy is only able to choose between $a \& e$ in this case; when there is a big gap in the \#47's service, it is able to choose to walk up to the streetcar rather than wait for a bus that is not forthcoming. Once it has opted to wait for the \#47 however, the strategy as we have defined it is locked into taking the itinerary with the best mean travel time which in this case is a.

Other OD pairs likely present itineraries where the real-time strategy is able to make more distinct choices, though in this case it plays a limited role. Though not modelled here, it may be the case that many actual passengers make use of real-time information throughout their trip, enabling them to select subsequent legs based on real-time information. For example it is quite easy in practice to choose between itineraries $a$ and $b$. The only difference between the two is in whether to walk or use transit for the final leg and the station where the choice must be made has a real-time arrival display and other visual cues.

\section{Results}

The main goal of this research is to demonstrate that more realistic route-choice assumptions generate travel time estimates which are substantially higher than those produced by standard shortest-path algorithms. To quantify this, Section 4.2 measures the shift toward longer travel time distributions associated with these strategies. To appreciate those results however it is helpful to first understand something of the complexity of the paths which would be required to achieve an optimal travel time for all departures in the first place. Thus Section 4.1 first provides measures of the path complexity associated with the sample of OD pairs. Finally, Section 4.3 further illustrates the choice situation, giving a passenger's perspective of the matter by measuring the potential upside travel time risk associated with route choice at any given moment of departure.

\subsection{Optimal Path Complexity}

Only a small portion of OD pairs in the sample produce only one itinerary across an entire service period. A hypothetical passenger on the TTC, intending to minimize their travel time, would thus face at least a couple viable options for most potential trips, whether or not they were strictly aware of these. Relative distributions of itinerary counts are given in Figure 5 separately for each of the four daytime service periods.

The distribution of itinerary counts is fairly similar throughout the day, though the midday and PM-peak service have somewhat longer tails. This corresponds to generally longer travel times and increased congestion relative to the AM-peak and evening periods. A typical (median) trip 


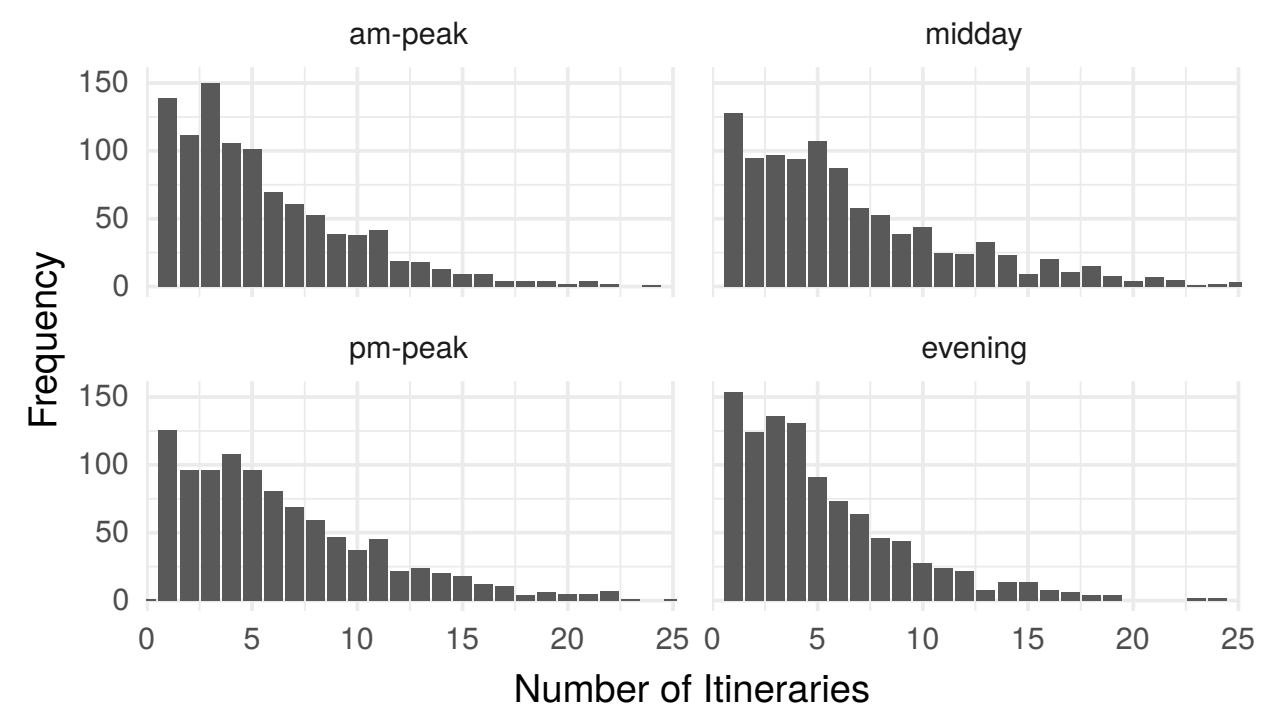

Figure 5: Histogram showing frequency of itinerary counts across OD pairs.

produces 5 distinct itineraries in a given service period which roughly matches our expectations and corresponds to the example in Section 3.6. The long tails of the distributions do come as somewhat of a surprise, with $10 \%$ of OD pairs producing 12 or more itineraries.

Such large counts however may perhaps only reflect the inclusion of itineraries which are only the optimal path for as little as a single minute in the departure window. The measure of entropy described in Section 3.4 is designed to account for this, factoring in consideration of how much time in the service period each of the set of itineraries is optimal. Figure 6 plots entropy per OD pair as a cumulative density function for each service period. Given that an entropy of 2 bits corresponds to a scenario with four equally likely outcomes and an entropy of 3 bits to 8 equally likely outcomes, we can see from the chart that optimal route-choice on the TTC can be extremely complex for some trips. Similar to Figure 5 we see generally similar patterns across the service periods, though with somewhat higher values in the midday and pm-peak periods. Note that the median value of $\approx 1.6$ again corresponds well with the example given in Section 3.6. It may be important to recall here what was shown in that example: that distinct itineraries can share a large portion of their route and that relatively minor differences in travel time can flip the optimal itinerary rapidly between a few different values. The last leg of a trip for example may be walked or rely on transit if a vehicle happens to be coming.

This high level of path complexity is concordant with the idea of the hyperpath presented by Schmöcker et al. (2009), though of course we question whether a typical passenger is capable of or interested in allowing such complexity to manifest in their actual travel behaviour. While not shown here, preliminary results indicate that a comparable entropy measure derived from a schedule-based GTFS package for the same period produced a similar distribution overall, though 


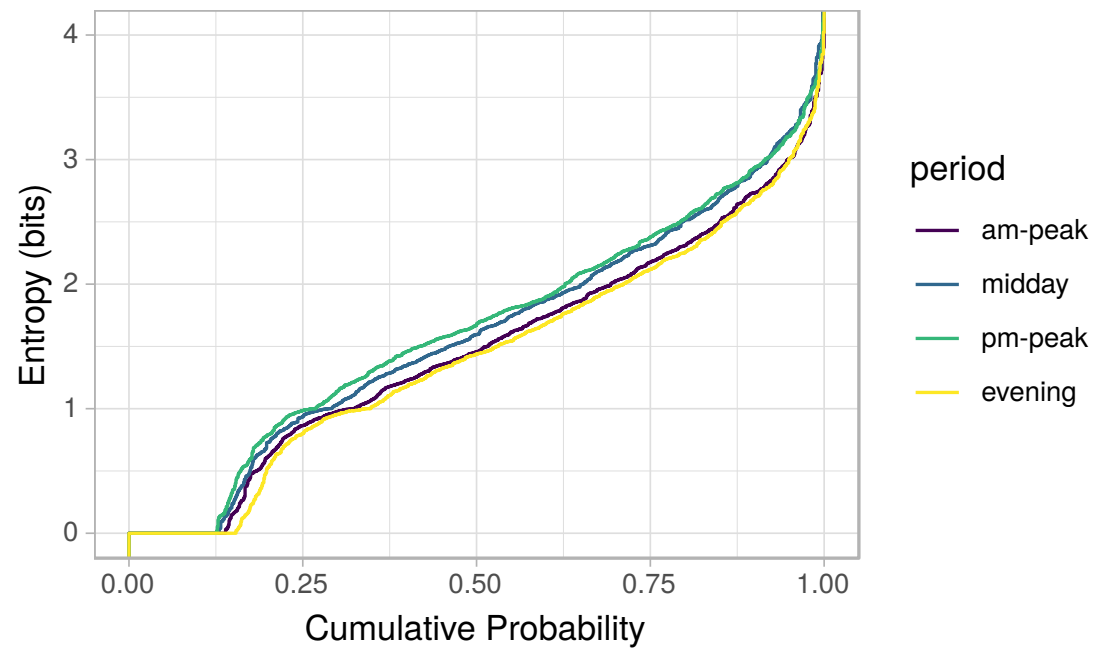

Figure 6: Cumulative distribution of $S$ per service periods.

with values shifted somewhat toward lower values of $S$. This is to be expected as the schedule data typically contains repeating patterns with little or no random variability in service.

$S$ is fairly stable for OD pairs across the four service periods with Pearson correlation values ranging from $r=0.87$ (AM-peak vs. midday) to $r=0.82$ (AM-peak vs. evening). Exploratory regression analysis was able to discover significant patterns in the distribution of $S$, showing that OD pairs with higher values of $S$ tend to be associated with longer average travel times and/or greater distances between origin and destination. They also tend to be oriented at a diagonal to the orthogonal street/transit grid of the city, thereby being more likely to require transfers as few direct diagonal services exist. OD pairs which would likely involve travel crossing a subway line also produced higher $S$ values, perhaps due to the use of subway stations as major transfer points in some places. Finally, OD pairs that happened to be aligned with well travelled corridors generally had somewhat lower values of $S$, which we speculate may be due to a streamlining or upgrading of service in busy corridors. To avoid burdening the reader with unnecessary detail here, a fuller description of the variables and regression results is offered in Appendix A.

While the a priori choice of a travel-time optimizing path on a particular trip may be daunting, it is entirely possible that the difference in travel times between the optimal and heuristic alternatives is actually quite negligible. This is especially true since the alternatives are based on the same set of itineraries and trips and thus different strategies may result in travel times identical to those of the optimal-choice scenario. Sections 4.2 and 4.3 explore the implications of the alternative route-selection heuristics for estimated travel times, first in terms of the shift in travel time distributions and then in terms of travel time risk associated with route choice at a moment of departure. 


\subsection{A Shift Toward Longer Travel Times}

Studies of transit accessibility are often based on distributions of travel times, summarizing accessibility over a particular time period such as the morning commute (e.g. Owen and Murphy, 2018a; Allen and Farber, 2018). To measure the potential overestimate of accessibility attributable to the use of shortest-path based estimates, we compare the distributions of travel times derived from the standard optimal-path model with those derived with the two alternative strategies defined in Section 3.5.

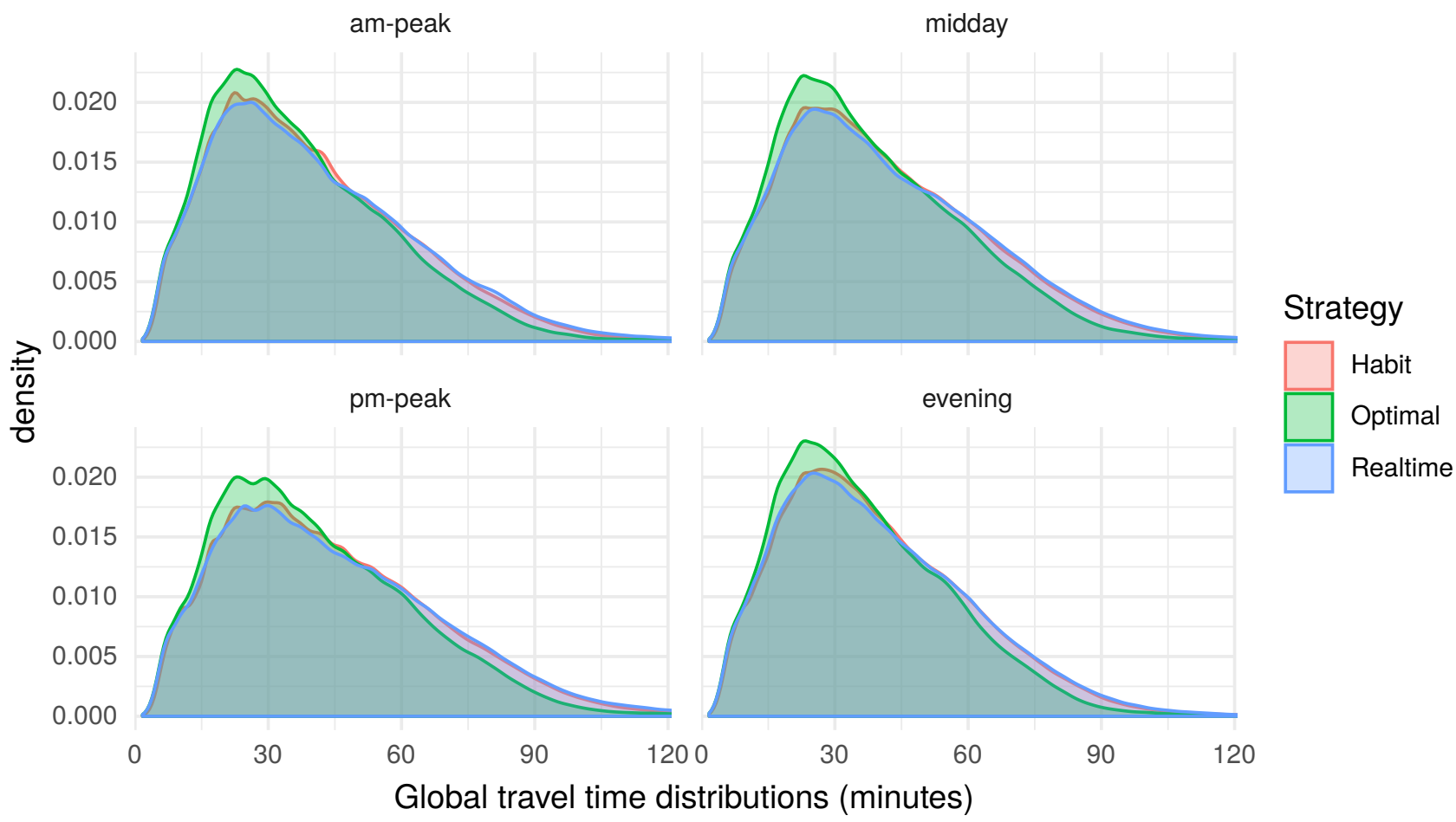

Figure 7: Overplotted distributions of all travel times for the three route-selection strategies per time period.

Figure 7 plots the global distribution of travel times across all sampled OD pairs for the three route-selection strategies. Our sample includes trips taking anywhere from several minutes to two hours or more and travel times are generally slightly longer in the midday and PM-peak periods. As expected the distributions for both heuristic strategies are seen to have shifted somewhat toward longer travel times relative to the standard measure. While this shift doesn't look like much visually, it is important to appreciate that Figure 7 covers a two hour period in order to show the whole range of travel times, making small relative differences difficult to notice. Across all four periods, median travel times for the habitual and realtime strategies are about 3 or 4 minutes longer respectively while at the $90^{\text {th }}$ percentile they are shifted 6 to 7 minutes or about $8-10 \%$ relative to the optimal-path estimates. Basic descriptive statistics of each distribution are 
given in Table 3.

Table 3: Descriptive statistics of the travel time distributions shown in Figure 7. Values are in minutes.

\begin{tabular}{c|rccc} 
Period & Strategy & Mean & Median & $90^{\text {th } \% \text { ile }}$ \\
\hline \multirow{4}{*}{ AM-peak } & Optimal & 37.2 & 33.5 & 65.5 \\
& Habit & 40.1 & 36.1 & 70.8 \\
& Real-time & 40.8 & 36.5 & 72.7 \\
\hline \multirow{4}{*}{ Midday } & Optimal & 38.5 & 34.7 & 67.2 \\
& Habit & 41.8 & 37.8 & 73.0 \\
& Real-time & 42.2 & 38.1 & 74.3 \\
\hline \multirow{4}{*}{ Evening } & Optimal & 40.7 & 37.0 & 71.1 \\
& Habit & 44.3 & 40.5 & 77.7 \\
& Real-time & 44.9 & 40.7 & 79.3 \\
\hline & Optimal & 36.7 & 33.5 & 63.3 \\
& Habit & 39.6 & 36.2 & 68.6
\end{tabular}

Just as $S$ varied substantially between OD pairs, we should expect to see a good deal of variability in travel time differences when we disaggregate these global statistics into summaries per OD pair and service period. Figure 8 shows distributions of relative difference in the median travel time per OD pair and time period. From this perspective we can see that some ODs have distributions shifted as much as 12 minutes toward longer travel times, even in the middle of their respective distributions, though more than half of OD pairs have median shifts of less than three minutes relative to the standard optimal-path distributions.

The realtime strategy seems to produce slightly longer travel times than the habitual strategy on average, and as in the previous plot it is interesting to note how similar the two heuristic methods are in their degree of difference from the optimal method. One possible explanation is that the real-time strategy as defined here can only distinguish between the first legs of potentially involved itineraries, defaulting after that to the safest itinerary using that first leg. It may be the case that this often just results in the same choice as the habitual strategy. While the relative distributions may look similar however, the two measures are only correlated at $r=0.56$.

As with $S$ in Section 4.1, we find that the heuristic travel time distributions both tend to be shifted further away from the optimal path scenario, in absolute terms, when trips are longer, oriented at an angle to the grid, crossing a subway line and in corridors less frequently travelled. The interaction with these variables is not terribly strong though, only explaining 18-19\% of the variation in median travel time differences. Precise details of the regression models used are 
provided in Appendix A.

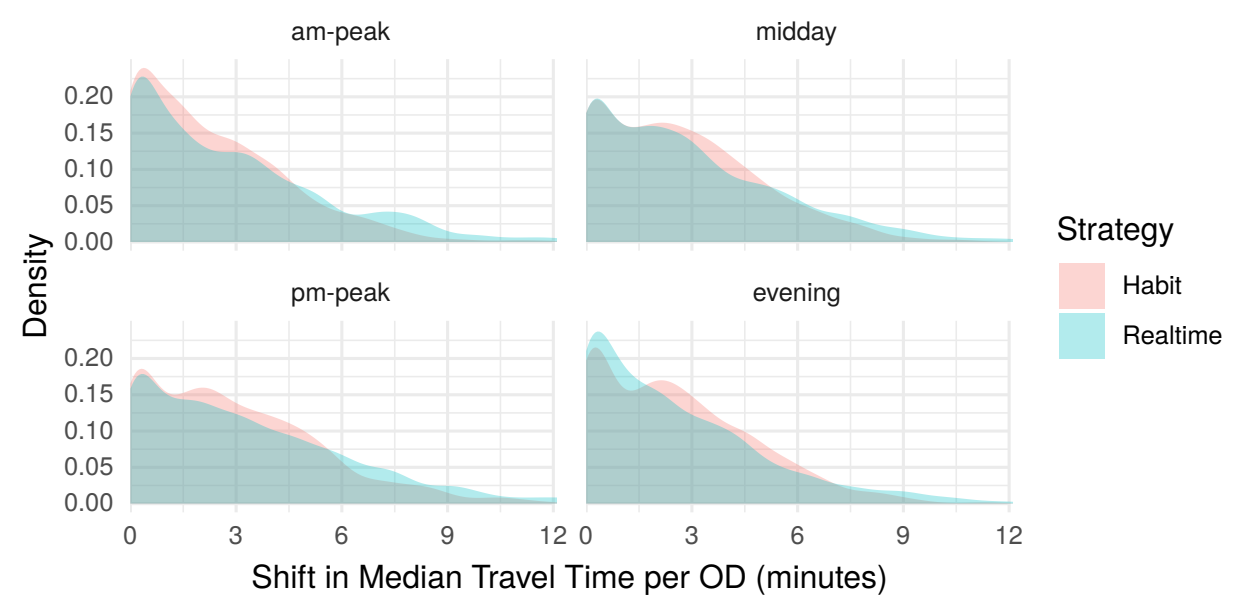

Figure 8: Distributions of difference in median travel times for each strategy relative to the standard optimal method. Observations are per OD pair and time period.

\subsection{Route Choice \& Travel Time Risk}

Another way to think about the difference in travel times attributable to route selection strategy is in terms of risk associated with route choice at a particular moment of departure. That is, when adopting a reasonable route-selection heuristic, what are the odds that there was actually a non-selected alternative that would have been much faster? This relates to the concept of "regret", which has been applied before in the context of route choice behaviour (Caspar, 2012; Ben-Elia, Ishaq, and Shiftan, 2013). Briefly, the idea is that people seek to make choices such that a nonselected alternative does not perform significantly better than the one chosen. In a sense, this is the inverse of risk aversion, pushing people to make risky choices to avoid losing out on potentially large gains. It also maps loosely onto the concept of "upside risk" in finance. Our interest in this metric in the context of this paper is that we would expect OD pairs with large upside risk to encourage passengers to engage in more dynamic strategies in an effort to optimize their travel times. With the data we have we can measure the extent of this phenomenon, though of course we do not attempt to associate our observations with actual passenger behaviour in this paper.

We find that in aggregate, across all ODs and times, the habitual strategy results in an optimal itinerary choice $59 \%$ of the time while the real-time strategy is able to do only slightly better at $60 \%$. Only a small percentage of OD pairs were able to achieve perfect travel time optimality at all times though more than half achieved optimal travel times for more than half of the time. To illustrate some of this variation, Figure 9 plots a distribution of the difference between the optimal and heuristic travel times for a random sub-sample of 100 OD pairs. Service periods did not show 
substantial differences and are aggregated together for this plot.

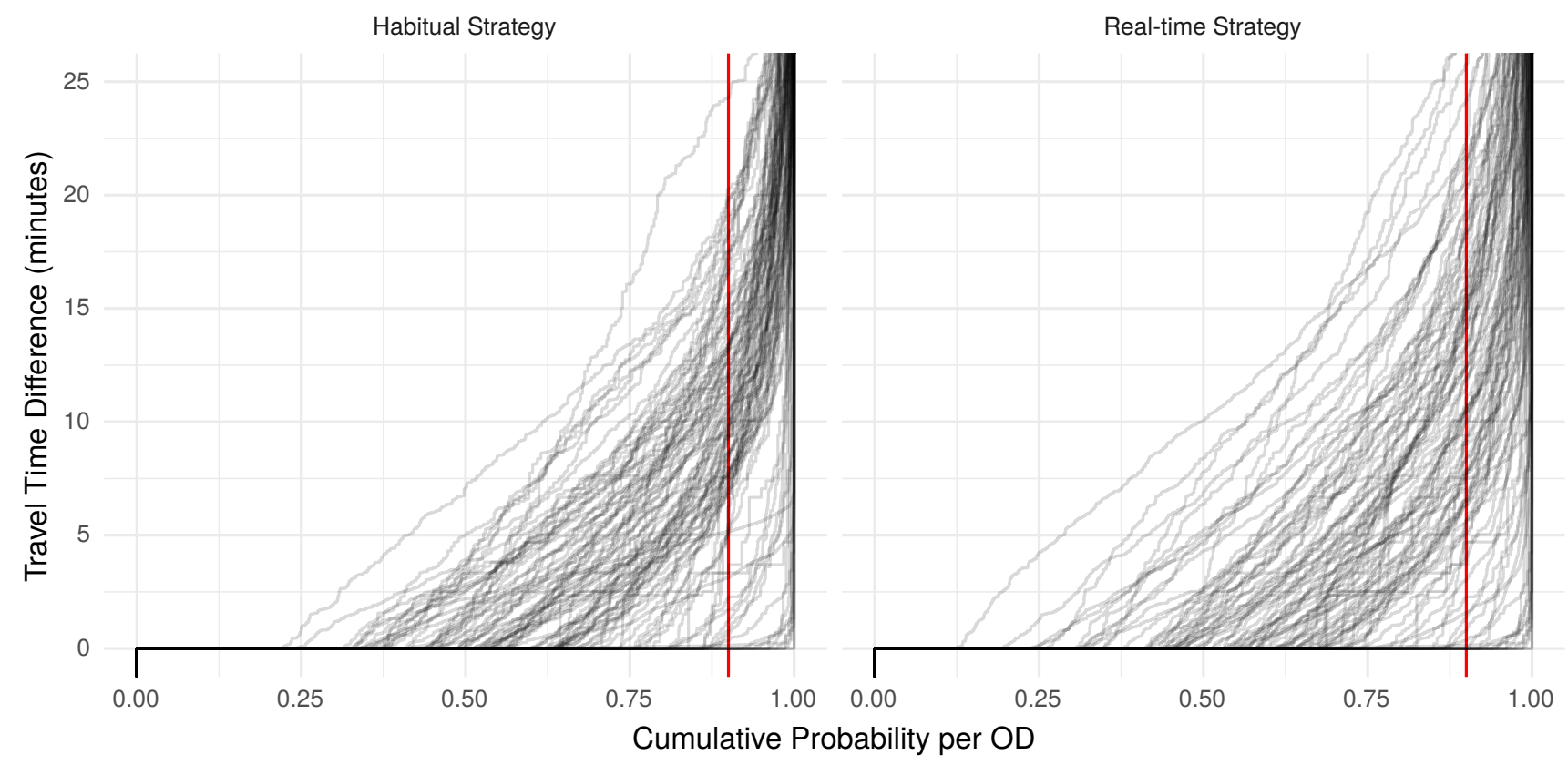

Figure 9: Distributions of difference in travel time for a given moment of departure. Each line represents one OD pair. The red line indicates the 90th percentile observation.

It seems clear that while a majority of departure times give equal values for the optimal and heuristic strategies, there is a decidedly long tail for many OD pairs where either heuristic strategy results in travel times 10 or even 20 or more minutes longer than was strictly necessary. In reality, some such trips, especially for shorter expected travel times, might induce passengers to change their strategy if possible. This can sometimes be seen in Toronto where passengers, after waiting longer than they would like will hail a cab, start walking to another stop, rent a bike, or otherwise leave their ordinary itinerary and make alternate arrangements. The effect is sometimes contagious, as one person talking with others and departing can trigger nearby passengers to question their willingness to continue waiting. It is important to consider however that the theoretical optimal time at any given moment is not something that passengers are actually privy to. What they can experience is a delay relative to an expected travel or wait time along with some knowledge that alternate paths might have been taken; if they have tried the alternatives, they may also have some knowledge of the likely travel times on these to compare their experience to.

One consistent pattern in Figure 9 is that the real-time strategy has slightly more extreme values at the tails; this may be the result of impatience regarding the first leg leading to bad choices in regard to total travel times. Recall also that the habitual strategy tends to prefer routes with few large outlying travel times. For both strategies almost all OD pairs in all service periods 
show increased travel times by the $90^{\text {th }}$ percentile.

\section{Conclusions and Discussion}

It has been noted in the literature that transit accessibility models may falsely assume that passengers have perfect knowledge of the transit system and are able to manifest this knowledge in their moment-to-moment route choice. This paper has spelled out the implications of this assumption and put it to an empirical test for one major transit agency see whether making more realistic assumptions about passenger route choice ends up producing reasonably similar results. The TTC was chosen because its large grid of frequent transit lines and questionable schedule adherence were expected to maximize the difference between the standard model and more realistic heuristic strategies by presenting passengers with complex and dynamic itineraries among which to choose. Section 4.1 showed that this expected route-choice complexity was quite real with passengers having multiple distinct itineraries to choose from for almost all OD pairs. Given the median optimal route-choice entropy of about 1.7, it's not surprising that almost half of departures modelled with the alternative route-selection strategies chose an itinerary that would end up being sub-optimal in terms of travel time, relative to the standard shortest-path model.

At a moment-to-moment level, we found that route-choice has serious implications for expected travel times, with a small portion of trips taking much longer than a path-indifferent travel-time estimate would predict. We describe this as the "travel time risk" associated with route choice for an OD pair and find it to be strongly related $\left(0.41 \geq r^{2} \geq 0.58\right)$ to the entropy of the timeoptimal path. Despite this potentially large difference in travel times given a particular moment of departure, we find that when travel times are considered as distributions over a time window, the shift toward longer travel times seems relatively small with the average (median) trip gaining less than 5 minutes at the 90th percentile. Again though, there appears to be a long tail with some OD pairs seeing large shifts in travel time distributions.

We attempted to identify characteristics of OD pairs that were related to greater route-choice entropy and greater shifts in travel time distributions, but were unable to explain more than about $20-25 \%$ of the variation with simple spatial measures. In general, longer trips and those likely to require transfers produced greater differences from the standard perfect-information route-choice model. To offer some explanation for the greater part of the variance would require further study.

To our knowledge, this study is the first to look at this phenomenon directly and much work remains to be done. It is important for example to consider that our definition of more realistic route-choice algorithms (Section 3.5) is far from a fully developed model of human behaviour. While denying transit passengers omniscience about their ultimate travel time, we still attribute to them too much capacity in some areas and too little in others. For example, the realtime 
strategy we defined has no ability to change course midway through a trip though it is easy to observe some actual passengers doing just this in response to delays. Conversely, the habitual strategy as we defined it seems optimistic, imagining that passengers have an accurate view of the mean travel times on all of their alternatives when it seems unlikely they would even know what all the alternatives are. Indeed, both strategies seem fanciful when we consider how much information actual passengers are likely have about trips to places they've never been before; this is especially relevant in the context of accessibility studies and needs much more consideration.

How much our findings can be extended to other agencies or to schedule-based data is somewhat unclear. The TTC is a large agency with most routes offering frequent service. Other agencies with different service patterns, for example radial routes and infrequent service, likely have much more constrained route choice for most trips, though perhaps the implications of choosing a sub-optimal route would be more dramatic. It may be useful to look at the problem in a slightly different way by noting that an abundance of alternative routes implies that the network is resilient to suboptimal route choice by passengers. By choosing to study a more "resilient" agency we may have failed to observe places where route choice has more substantial consequences.

As for the use of schedule-based GTFS data in transit accessibility models, we can only suggest that route choice complexity will appear diminished and that travel times will tend to exhibit less variability overall. Previous research has suggested that schedule-based GTFS data has serious limitations in its ability to accurately model transit services as people actually experience them, producing potentially large overestimates of access and systematic spatial errors (Wessel and Farber, 2019; Stewart, 2017). While schedule-based analysis is likely to continue into the near future due to the availability of data, we would suggest that more realistic models are needed both of route-choice behaviour and of on-the-ground transit fleet operations.

This study while not itself conclusive is, we hope, suggestive of directions which could be taken in future research on accessibility. As transit operations are modelled with a higher degree of detail and thus typically a stronger presumption of precision, it becomes necessary to consider how precise it is possible to be while building a model of reality in which human agency and human limitations play an active part.

\section{References}

Allen, Jeff and Steven Farber (2018). Generating measures of access to employment for Canada's eight largest urban regions. Tech. rep. University of Toronto. URL: https: //osf .io/preprints/ socarxiv/pvrd9/. 
Ben-Elia, Eran, Robert Ishaq, and Yoram Shiftan (2013). "'If only I had taken the other road...": Regret, risk and reinforced learning in informed route-choice". In: Transportation 40.2, pp. 269293.

Blanchard, Samuel and Paul Waddell (2017). "Generalized Methodology for Measuring Regional accessibility with an Integrated Pedestrian and Transit Network". In: Transportation Research Record.

Boisjoly, Geneviève and Ahmed El-Geneidy (2016). "Daily fluctuations in transit and job availability: A comparative assessment of time-sensitive accessibility measures". In: Journal of transport geography 52, pp. 73-81.

Caspar, G (2012). "Regret theory-based route choices and traffic equilibria". In: Transportmetrica 8.4, pp. 291-305.

Conway, Matthew Wigginton, Andrew Byrd, and Marco van der Linden (2017). "Evidence-based transit and land use sketch planning using interactive accessibility methods on combined schedule and headway-based networks". In: Transportation Research Record: Journal of the Transportation Research Board 2653, pp. 45-53.

Cui, Mengying and David Levinson (2018). "Full cost analysis of accessibility". In: Journal of Transport and Land Use 11.1.

Delling, Daniel, Thomas Pajor, and Renato F Werneck (2014). "Round-based public transit routing". In: Transportation Science 49.3, pp. 591-604.

Dunlap, Matthew et al. (2016). "Estimation of origin and destination information from Bluetooth and Wi-Fi sensing for transit". In: Transportation Research Record: Journal of the Transportation Research Board 2595, pp. 11-17.

Eluru, Naveen, Vincent Chakour, and Ahmed El-Geneidy (2012). "Travel mode choice and transit route choice behavior in Montreal: insights from McGill University members commute patterns". In: Public Transport 4.2, pp. 129-149.

Farber, Steven and Liwei Fu (2017). "Dynamic public transit accessibility using travel time cubes: Comparing the effects of infrastructure (dis)investments over time". In: Computers, Environment and Urban Systems 62, pp. 30-40.

Gärling, Tommy (1998). "Behavioural assumptions overlooked in travel-choice modelling". In: Travel Behaviour Research: Updating the State of Play, pp. 1-25.

Gärling, Tommy and Kay W Axhausen (2003). "Introduction: Habitual Travel Choice". In: Transportation 30.1, pp. 1-11.

El-Geneidy, Ahmed et al. (2016). "The cost of equity: Assessing transit accessibility and social disparity using total travel cost". In: Transportation Research Part A: Policy and Practice 91, pp. 302-316.

Gray, Robert M (2011). Entropy and information theory. Springer Science \& Business Media. 
Jang, Wonjae (2010). "Travel time and transfer analysis using transit smart card data". In: Transportation Research Record: Journal of the Transportation Research Board 2144, pp. 142-149.

Jánošíková, L’udmila, Jiří Slavík, and Michal Koháni (2014). "Estimation of a route choice model for urban public transport using smart card data". In: Transportation planning and technology 37.7, pp. 638-648.

Kaplan, Sigal et al. (2014). "Using connectivity for measuring equity in transit provision". In: Journal of Transport Geography 37, pp. 82-92.

Kim, Jiwon, Jonathan Corcoran, and Marty Papamanolis (2017). "Route choice stickiness of public transport passengers: Measuring habitual bus ridership behaviour using smart card data". In: Transportation Research Part C: Emerging Technologies 83, pp. 146-164.

Kurauchi, Fumitaka, Jan-Dirk Schmöcker, Achille Fonzone, et al. (2012). "Estimating weights of times and transfers for hyperpath travelers". In: Transportation Research Record 2284.1, pp. 89-99.

Kurauchi, Fumitaka, Jan-Dirk Schmöcker, Hiroshi Shimamoto, et al. (2014). "Variability of commuters' bus line choice: an analysis of oyster card data". In: Public Transport 6.1-2, pp. 2134.

Lee, Haengju et al. (2017). "Metrotime: Travel time decomposition under stochastic time table for metro networks". In: Smart Computing (SMARTCOMP), 2017 IEEE International Conference on. IEEE, pp. 1-8. URL: https://ieeexplore.ieee.org/abstract/document/7947021.

Lee, Jinhyung and Harvey J Miller (2018). "Measuring the impacts of new public transit services on space-time accessibility: An analysis of transit system redesign and new bus rapid transit in Columbus, Ohio, USA". In: Applied Geography 93, pp. 47-63.

Ma, Ting and Gerrit Jan-Knaap (2014). "Analyzing employment accessibility in a multimodal network using GTFS: a demonstration of the purple line, Maryland". In: The Association of Collegiate Schools of Planning (ACSP) Annual Conference, Philadelphia, Pennsylvania.

Morang, Melinda (2019). Documentation for "Add-GTFS-to-a-network-dataset". URL: https : // github . com/Esri / public-transit-tools / blob/master / add-GTFS - to-a-networkdataset/README.md.

Nassir, Neema et al. (2016). "A utility-based travel impedance measure for public transit network accessibility". In: Transportation Research Part A: Policy and Practice 88, pp. 26-39.

OpenTripPlanner Project (2019). OpenTripPlanner on Github. URL: https : / / github . com / opentripplanner/OpenTripPlanner.

Owen, Andrew and David Levinson (2016). "Developing a comprehensive US transit accessibility database". In: Seeing Cities Through Big Data. Springer, pp. 279-290.

Owen, Andrew and Brendan Murphy (2018a). "Access Across America: Transit 2017". In: URL: http://hdl . handle.net/11299/199920. 
Temporal Sampling and Service Frequency Harmonics in Transit Accessibility Evaluation (2018b). Transportation Research Board. URL: https://trid.trb.org/view/1497217.

Schmöcker, Jan-Dirk et al. (Jan. 1, 2009). "A Game Theoretic Approach to the Determination of Hyperpaths in Transportation Networks". In: Transportation and Traffic Theory 2009: Golden Jubilee. Springer. ISBN: 978-1-4419-0819-3. DOI: 10 . 1007/978-1-4419-0820-9_1. URL: http://dx.doi.org/10.1007/978-1-4419-0820-9_1.

Stenneth, Leon et al. (2011). "Transportation mode detection using mobile phones and GIS information". In: Proceedings of the 19th ACM SIGSPATIAL International Conference on Advances in Geographic Information Systems. ACM, pp. 54-63.

Stewart, Anson F. (2017). "Advancing accessibility: public transport and urban space". PhD thesis. Massachusetts Institute of Technology. URL: http://hdl . handle.net/1721.1/111444.

Sun, Yanshuo and Ruihua Xu (2012). "Rail transit travel time reliability and estimation of passenger route choice behavior: Analysis using automatic fare collection data". In: Transportation Research Record: Journal of the Transportation Research Board 2275, pp. 58-67.

Toronto Transit Commission (2019). Service Details. URL: https : / / www . ttc . ca/Routes / General_Information/Service_Details.jsp.

TTS (2019). Transportation Tomorrow Survey. URL: http://www.transportationtomorrow.on. $\mathrm{ca} /$.

Wessel, Nate, Jeff Allen, and Steven Farber (2017). "Constructing a Routable Retrospective Transit Timetable from a Real-time Vehicle Location Feed and GTFS". In: Journal of Transport Geography 62, pp. 92-97. URL: http://sausy.ca/wp-content/uploads/2017/11/retroGTFS-paper .pdf.

Wessel, Nate and Steven Farber (2019). "On the Accuracy of Schedule-Based GTFS for Measuring Accessibility". In: DOI: 10 . 31235 / osf . io/hzgpd. URL: https : / / osf . io/preprints / socarxiv/hzgpd/.

Xue, Yuan et al. (2011). Empirical characteristics of transit travel time distribution for commuting routes. Tech. rep. URL: https://trid.trb.org/view/1092692.

Zervaas, Quentin (Oct. 23, 2018). TransitFeeds.com. URL: https://transitfeeds.com/. 


\section{A Characterizing OD Pairs}

This section discusses in detail the regression models covered only briefly in Sections 4.2 \& 4.3.

Given the variability which exists between OD pairs on measures of entropy and travel time difference, one may rightly ask what qualities of an OD pair are associated with higher or lower values. Are there spatial patterns? My approach to answering these questions was to conduct some exploratory regression analysis to see how well the variables of interest can be predicted and what attributes are positively or negatively associated. I produced a number of simple OD-level variables, listed below, based on the origin and destination locations. A few other variables were tried but found to be unrelated and are not listed here.

Table 4: Description of regression variables.

\begin{tabular}{l|l} 
Variable & Description \\
\hline grid_dist & $\begin{array}{l}\text { Manhattan distance in kilometers between origin and } \\
\text { destination accounting for the orientation of Toronto's } \\
\text { street grid }\end{array}$ \\
\hline from_grid & $\begin{array}{l}\text { Minimum angle between the line formed by the OD pair } \\
\text { from the street grid of Toronto, which is defined as an } \\
\text { orthogonal axis rotated 17 degrees counterclockwise from } \\
\text { true north. }\end{array}$ \\
\hline crosses_sub & $\begin{array}{l}\text { Boolean variable indicating whether a straight line con- } \\
\text { necting the origin and destination crosses one of the sub- } \\
\text { way lines }\end{array}$ \\
\hline real_flow & $\begin{array}{l}\text { Approximation of actual daily transit travel demand be- } \\
\text { tween origin and destination based on data from the 2016 } \\
\text { Toronto Travel Survey. }\end{array}$ \\
\hline max_from_sub & $\begin{array}{l}\text { Maximum distance in kilometers of either origin or des- } \\
\text { tination from the nearest subway line }\end{array}$
\end{tabular}

My initial assumption was that trips would be more complex and harder to optimize with an heuristic strategy when they are longer and at greater angles relative to the street grid. The reason for this is that longer trips will tend to present more opportunities for transfers to other lines and that trips at an angle to the grid will require transfers due to the generally orthogonal structure of the transit network in the city. Trips on the diagonal can generally either go "up-and-over" or "over-and-up", presenting at least two alternatives. I also suspected that OD pairs relying heavily on the subway would tend to produce fewer options and more similar travel times due to 1) the fact that this part of the system was modelled with schedule data and was thus artificially 
reliable and 2 ) the extremely short headways ( $\approx 3$ minutes) and reasonably fast travel on these lines were expected to dominate nearby alternatives. Finally, I expected OD pairs with fewer actual passengers would tend to have less reliable service on the assumption that the TTC would prioritize heavily travelled routes with e.g. express services or designated rights of way.

The analysis presented here is exploratory in spirit and meant primarily to suggest relationships with variables to help the reader understand some potential causes of route-choice complexity (measured with $S$ ) and differences in estimated travel time outcomes which can be measured in a variety of ways. The intention is not to offer generalizable evidence of any kind as many of these variables will have little relevance in the context of other transit agencies. I focus rather on the degree to which the variables of interest can be explained by the initial hypotheses. Rather than conducting a separate analysis for each time period, these are combined together in the same models. To the extent that some of the variation in $S$, etc. exists between time periods, some of that variation will not be explainable by these models.

The results of regressing $S$ (See Equation 1) on the variables in Table 4 are given in Table 5 below. The expected signs are seen for variables except for those to do with the subway: crosses_sub which is positive and max_from_sub which is insignificant. That crosses_sub indicates a positive relationship may mean that crossing the subway is positively associated with the complexity of the optimal path, $S$. This contradicts expectations but may reflect the fact that subway stations are often transfer points serviced by multiple lines. Roughly $26 \%$ of the variance is explained by the model.

Table 5: Regression predicting $S$ per OD pair and time period. $N=4,000, r^{2}=0.26$

\begin{tabular}{l|r|c} 
variable & $\beta$ & $p$ \\
\hline intercept & 0.715 & $<2 \mathrm{e}-16$ \\
grid_dist & 0.052 & $<2 \mathrm{e}-16$ \\
from_grid & 0.016 & $<2 \mathrm{e}-16$ \\
crosses_sub & 0.248 & $2.19 \mathrm{e}-13$ \\
real_flow & -0.004 & $1.70 \mathrm{e}-06$ \\
max_from_sub & -0.007 & 0.245
\end{tabular}

The same independent variables are now applied to a model of the difference in median travel times between the heuristic strategies and the optimal-path method; the distribution of this variable was shown in Figure 8. Table 6 gives results for the habitual strategy and Table 7 for the realtime strategy.

Regressions on other travel-time-related variables produced very similar results, including models for differences in mean travel times and travel times at the $90^{\text {th }}$ percentile. Except for the 
Table 6: Regression predicting difference in median travel times for the habitual strategy per OD pair and time period. $N=4,000, r^{2}=0.19$

\begin{tabular}{l|r|c} 
variable & $\beta$ & $p$ \\
\hline intercept & 1.023 & $<2 \mathrm{e}-16$ \\
grid_dist & 0.114 & $<2 \mathrm{e}-16$ \\
from_grid & 0.022 & $<2 \mathrm{e}-16$ \\
crosses_sub & 0.254 & 0.00241 \\
real_flow & -0.013 & $2.53 \mathrm{e}-10$ \\
max_from_sub & 0.048 & 0.00100
\end{tabular}

Table 7: Regression predicting difference in median travel times for the real-time strategy per OD pair and time period. $N=4,000, r^{2}=0.20$

\begin{tabular}{l|r|c} 
variable & $\beta$ & $p$ \\
\hline intercept & 0.688 & $1.59 \mathrm{e}-06$ \\
grid_dist & 0.156 & $<2 \mathrm{e}-16$ \\
from_grid & 0.027 & $<2 \mathrm{e}-16$ \\
crosses_sub & 0.443 & $5.59 \mathrm{e}-05$ \\
real_flow & -0.013 & $1.44 \mathrm{e}-06$ \\
max_from_sub & 0.085 & $7.79 \mathrm{e}-06$
\end{tabular}

changing influence of max_from_sub, all three models show similar relationships, reflecting the fact that $S$ is strongly correlated with any measure of difference in travel times. For the models given here, $S$ has a Pearson correlation with the difference in median travel time of 0.78 for the habitual strategy and 0.61 for the realtime. In part, this relationship is baked into the definition of the heuristic alternative strategies, which will almost certainly choose the sub-optimal itinerary more often when the information content of the optimal path is higher. This however may be a necessary feature of any possible heuristic route-selection strategy. If that is the case then an estimate of $S$ associated with a given trip might reasonably be used as a way of inflating travel time estimates derived from a standard shortest-path routing algorithm, at least until better algorithms are developed. For the TTC, a 1 bit increase in $S$ was associated with a roughly 1.8 minute increase in estimated travel time at the median for the habitual strategy and 1.9 minutes for the realtime strategy. This coefficient would however likely be very different for different agencies. 